\title{
The Most Frequent Prescription in Obstetrics: Bed Rest!
}

\author{
Harkiran Kaur Khaira and Smita Sinha* \\ Dept of OBG, Adesh Institute of Medical Sciences and Research (AIMSR). Bathinda, India
}

\begin{abstract}
The most frequent prescription across all medical disciplines and specially in Obstetrics has been bed rest. Even in this age of evidence based medicine, bed rest remains as the most prescribed, although evidence does not support it. The usage is strong because there are no substantial randomised trials to either refute or support bed rest, beginning with lack of definition. In this article we are reviewing bed rest in early miscarriage, hypertensive disorders of pregnancy, multiple pregnancy, preterm, reduced AFI, impaired fetal growth and in pregnancy after artificial reproduction techniques. We found that while women with moderate risk to preeclampsia, low AFI or multiple gestation may demonstrate some beneficial effects, it largely remains a tool for enforcing psychological benefit. It also entails adverse effects like muscle atrophy, stress and economic loss. However till more evidence can be made available, it will retain its numero uno position.
\end{abstract}

Keywords: Bed Rest, Preeclampsia, Preterm, Miscarriage, Reduced AFI, Multiple Pregnancy, ART

\section{Introduction}

Bed rest in pregnancy has been one of the most consistent and frequent prescriptions by Obstetricians the world over for the management of almost any deviation from normal gestation. In the common parlance it is advised by some doctors, even in a normal setting. Antepartum bed rest is advised based on the assumption that it might be both effective and safe for the mother and the fetus[1]. On many occasions, it is prescribed without sound evidence to its utility and sometimes just to allow the benefit of doubt to the expectant mother.

\section{Defining Bed Rest}

Across medical disciplines, bed rest would easily sum up to be the most popular prescription. However the term has never been defined accurately. Another term used often interchangeably is activity restriction, which leads to some confusion. Aerospace studies use the term bed rest to mean muscle disuse. Maloni JA[1] in her study defined antepartum bed rest as confinement to bed in hospital with activity limited to toileting. With this definition they observed that a pregnant woman spends about twenty-two hours in bed per day. In Obstetric practice, bed rest has often also involved instructions to use bed pan facility by the patient. The practice has been variant depending on clinical situation. Certainly a woman with minimal spotting per vagina in early pregnancy will be advised 'activity restriction' mostly but not completely limited to allowing toileting only; while a threatened preterm patient with 2 $\mathrm{cm}$ cervical dilatation and bag of membranes bulging will be ordered to use bed pan. There is a lack of randomised controlled trials to define what constitutes optimum antepartum bed rest.

\section{Bed rest in early miscarriage}

One in five pregnancies is complicated by vaginal bleeding before 20 weeks gestation and bed rest is routinely recommended[2]. It is prescribed based on the idea that as hard work and hard physical activity during pregnancy are associated with miscarriage, bed rest might reduce the risk[3]. However, this hypothesis is limited by the fact that most of the causes of miscarriage are not related to physical activity.

A survey among practitioners was conducted in Wessex, UK, 1985-86 and results published on what doctors thought about bed rest. It was thought to be usually ineffective by $413(32 \%)$ of the respondents; $623(48 \%)$ said that it was usually useful, while only $220(17 \%)$ thought that it was mandatory[4]. A resonating thought published in this study was that, many practitioners' believed that if they did not recommend bed rest, they might be held responsible for a subsequent miscarriage by the patient.

In a Cochrane review in 2010, only two studies including total 84 women were identified. There was no statistically significant difference in the risk of miscarriage in the bed rest group versus the no bed rest group (placebo or other treatment) (risk ratio RR 1.54, 95\% confidence interval CI 0.92 to 2.58)[5]. There was a higher risk of miscarriage in those women in the bed rest group than in those in the human chorionic gonadotrophin therapy group with no bed rest (RR 2.50, 95\% CI 1.22 to 5.11). However the analysis remains inconclusive due to small number of participants. Although there is no definite evidence that bed rest can affect the course of pregnancy, abstinence from active environment for a couple of days may help women feel safer, thus providing emotional relief[6]. 


\section{Bed rest in hypertensive disorders of pregnancy}

It is common practice to advice bed rest to women with preeclampsia. Restriction of activity and rest has even been advocated for prevention of hypertension in pregnancy[7]. Two small trials (145 women total) compared bed rest with ambulation in women with preeclampsia and found no conclusive evidence to accept or refute it[8]. Randomised trials by Spinapolice 1983 and Herrera 1993, evaluated risk reduction in preeclampsia of women who rested for four to six hours per day compared to women who carried out normal activity. One trial showed that there was a statistically significant reduction in the relative risk of pre-eclampsia with rest (relative risk RR 0.05, 95\% confidence interval, CI 0.00 to 0.83 ), however no risk reduction was observed for gestational hypertension[9]. In the second trial, rest of 30 minutes per day plus nutritional supplementation was associated with a reduction in the risk of pre-eclampsia ( 74 women; RR $0.13,95 \%$ CI 0.03 to 0.51 ) and also of gestational hypertension (RR $0.15,95 \%$ CI 0.04 to 0.63 )[10]. While these studies reflect a positive impact of bed rest on preventing preeclampsia, there is no information regarding perinatal or maternal outcome, as also bias in these studies cannot be completely ruled out.

While literature does not support bed rest as part of management protocol for preeclampsia, there appears to be a small but indefinite role in prevention of preeclampsia in women who are at moderate risk.

\section{Bed rest in multiple pregnancy}

Women and infants of a multiple pregnancy are recognised to be at increased risk of adverse outcome when compared with singletons. The greatest risk to infants of a multiple pregnancy is being born preterm[11]. The preterm birth rate less than 37 weeks for women with a singleton pregnancy is $6.3 \%$ and $97 \%$ for women with a triplet pregnancy[12]. Multiple pregnancies account for $9 \%$ to $12 \%$ of all perinatal deaths[13]. In addition, multiple births are associated with maternal complications such as preeclampsia, gestational diabetes, caesarean delivery, and postpartum haemorrhage[14]. The common prescription of bed rest is justified with the assumption that it effectively prevents preterm birth by reducing uterine activity and that bed rest is safe for mother and infants[1]. A Cochrane review on bed rest published in 2010, identified seven trials involving 713 women with multiple pregnancy. It was reported that routine bed rest in hospital did not improve the rates of preterm birth or perinatal mortality. There was a suggestion of a decreased number of low birthweight infants (less than $2500 \mathrm{~g}$ ) born to women in the routinely hospitalised group (RR 0.92; 95\% CI 0.85 to 1.00)[15]. For women with an uncomplicated twin pregnancy the results of this review show no benefit from routine hospitalisation for bed rest.

Evidence does not support routine prescription of bed rest in all women with multi fetal gestation, as it does not seem to improve preterm birth or perinatal mortality. There is no advantage in prevention of maternal complications as well. But with the slight advantage of improving birth weight of foetus, it may be beneficial in reducing the problems of preterm baby. Further trials are still warranted to accept or refute the small evidence.

\section{Bed rest in preterm}

Preterm birth can be due to a multitude of factors including chronic maternal illness, vaginal infections, stress, socio economic factors etc. Extremely few trials have been conducted evaluating bed rest in prevention of preterm birth. Elliot JP et al[16] conducted a randomised trial assessing the efficacy of activity restriction (AR) in preventing preterm birth in women who were negative for fetal fibronectin (fFN). A total of 73 women with negative fFN were randomised ( 36 with AR, 37 without AR). The overall preterm birth rate was $40 \%$, with $44.4 \%$ of patients with $\mathrm{AR}$ and $35.1 \%$ of patients without $\mathrm{AR}$ delivering preterm, $p=0.47816$. In an earlier study by Hobel CJ et al bed rest was addressed as a secondary outcome and no benefit was reported. However this study lacked in proper randomisation information and several important details like characteristics of women included in the study[17].

Thus evidence does not support activity restriction or bed rest as a valid intervention in preterm birth prevention. Again a safe conclusion is fugitive in the absence of trials. Hence though not evidenced, it cannot yet be refuted with a certainty.

\section{Bed rest in impaired fetal growth and reduced AFI}

Bed rest during pregnancy is one of the numerous approaches apart from nutritional supplementation, oxygen therapy, plasma volume expansion, beta mimetic drugs suggested to treat impaired fetal growth. We came across a single trial by Laurin in 1987, comparing birth weight among women on bed rest with ambulant but off work women. It was a small study involving 107 pregnant women, which reported that no differences were detected between bed rest and ambulatory management for fetal growth parameters (relative risk $0.43,95 \%$ confidence interval: 0.15 to 1.27 ) and neonatal outcomes[18]. The study was too small to be accepted as concrete evidence for medical advice. 
In a prospective study conducted in 2011, maternal rest in the left lateral decubitus position was shown to increase the estimated fetal urine production rate and the estimated amniotic fluid volume[19]. In another publication 2012, the principle finding reported was that the estimated amniotic fluid volume increase with maternal rest in the left lateral decubitus position follows the pattern of a saturation curve. The increase is maximal at the beginning; however, it is saturated as the position is maintained. Finally, the amniotic fluid volume increase stops at approximately at the second hour of rest. The maximum increase is in the first fifteen minutes[20]. It may be because the maternal left lateral position, having the least disturbing effect on fetal hemodynamics compared with any other position,[21,22] may increase uterine and placental blood flow. Therefore an apt prescription of bed rest in low AFI must include the instructions for rest in left lateral with rest limited to a short periods. Longer durations of rest is neither warranted nor effective.

\section{Bed rest after ART}

Women who have conceived with artificial reproductive techniques come tagged with an indefinite prescription for best rest, lest the 'precious' pregnancy be lost. While those who have undergone IUI (intra uterine insemination) may be less prone to resign to complete bed rest, those who have undergone IVF (in vitro fertilisation) are usually greatly stressed and resort to bed rest almost during the entire duration of gestation. Psychological it gives them a leverage in fighting the odds to a successful pregnancy outcome. Clinically it is one aspect that is still not well established; whether or not there are ways to decrease the movement or expulsion of embryos from the uterus following transfer. There are several studies evaluating effectiveness of post-ET (embryo transfer) techniques for women undergoing IVF and intracytoplasmic sperm injection (ICSI). The ongoing pregnancy rate was reported in one trial that compared immediate ambulation after embryo transfer to 30-minute bed rest. The results showed no evidence of an effect of bed rest in improving the rate of ongoing pregnancies (OR 1.00; 95\% CI 0.54 to 1.85 )[23].

\section{Adverse effects of bed rest}

Prolongedbedrestis fraughtwith potential problems. Bedrest may increase the likelihood of thromboembolic events[24], muscle atrophy and symptoms of musculoskeletal and cardiovascular de conditioning[25,26] may be stressful and costly for women and their family[27], may induce self blame feelings in case of failure to comply with the prescription[28] and may increase costs for the health services[29]. A study, conducted under highly standardised conditions, provides prospective evidence that 60 days of bed rest accelerated fat accumulation in the vertebral bone marrow of 24 healthy premenopausal women. The accumulation was not reversed 1 yr after a return to normal activities and was associated with normalised haemoglobin with decreased erythropoietin levels and with persistently elevated leukocyte numbers[30].

\section{Conclusion}

Bed rest even though is the most popular and maximally used prescription, lacks evidence to its utility. While women with moderate risk to preeclampsia, low AFI or multiple gestation may demonstrate some beneficial effects, it largely remains a tool for enforcing psychological benefit. However it demands to be practised with caution and appropriate titration as prolonged bed rest can lead to several complications.

\section{References}

1. Maloni JA. Lack of evidence for prescription of antepartum bed rest. Expert Rev Obstet Gynecol. 2011 July 1; 6(4): 385-393.

2. Sotiriadis A, Papatheodorou S, Makrydimas G. Threatened miscarriage: evaluation and management, BMJ 2004;329:152-5.

3. Lapple M. Occupational factors in spontaneous miscarriage. Zentralblatt fur Gynakologie 1990;112(8):457-66.

4. Everett C, Ashurst H, Chalmers I. Reported management of threatened miscarriage by general practitioners in Wessex. BMJ 1987; 295: 583-6

5. Aleman A, Althabe F, Belizán J, Bergel E. Bed rest during pregnancy for preventing miscarriage. Cochrane Database Syst Rev. 2005 Apr 18;(2):CD003576. [PMID: 15846669]

6. al-Sebai MA, Diver M, Hipkin LJ. The role of a single free beta-human chorionic gonadotrophin measurement in the diagnosis of early pregnancy failure and the prognosis of fetal viability. Hum Reprod 1996;11:881-8

7. Goldenberg R, Cliver S, Bronstein J, Cutter G, Andrews W, Mennemeyer S. Bed rest in pregnancy. Obstetrics \& Gynecology 1994;84:131-6.

8. Duley L. Strict bed rest for proteinuric hypertension in pregnancy. In: The Cochrane Library, Issue 2, 1995

9. Spinapolice RX, Feld S, Harrigan JT. Effective prevention of gestational hypertension in nulliparous women at high risk as identified by the rollover test. American Journal of Obstetrics and Gynecology 1983;146:166-8.

10. Herrera JA. Nutritional factors and rest reduce pregnancy induced hypertension and pre-eclampsia in positive rollover test primigravidas. International Journal of Gynecology \& Obstetrics 1993;41:31-5

11. Dodd JM, Crowther CA. Hospitalisation for bed rest for women with a triplet pregnancy: an abandoned randomised controlled trial and meta-analysis, BMC Pregnancy and Childbirth 2005, 5:8 
12. Australian Institute of Health and Welfare, National Perinatal Statis- tics Unit: Australia's mothers and babies 2000. Canberra 2003.

13. Norwitz ER, Edusa V, Park JS. Maternal physiologyand complications of multiple pregnancy. Seminars in Perinatology 2005;29(5):338-48. [PUBMED: 16360493]

14. Senat MV, Ancel PY, Bouvier-Colle MH, Breart G. How does multiple pregnancy affect maternal mortality and morbidity?. Clinical Obstetrics and Gynecology 1998;41(1): 78-83. [PUBMED: 9504226]

15. Crowther CA, Han S. Hospitalisation and bed rest for multiple pregnancy. Cochrane Database of Systematic Reviews 2010, Issue 7. Art. No.: CD000110. DOI: 10.1002/14651858. CD000110.pub2 [PMID:20614420]

16. Elliott JP, Miller HS, Coleman S, Rhea D, Abril D, Hallbauer K, Istwan NB, Stanziano GJ. A randomized multicenter study to determine the efficacy of activity restriction for preterm labor management in patients testing negative for fetal fibronectin. J Perinatol. 2005 Oct;25(10):626-30

17. Hobel CJ1, Ross MG, Bemis RL, Bragonier JR, Nessim S, Sandhu M, Bear MB, Mori B. The West Los Angeles Preterm Birth Prevention Project. I. Program impact on high-risk women. Am J Obstet Gynecol. 1994 Jan;170(1 Pt 1):54-62.

18. Laurin J, Persson PH. The effect of bedrest in hospital on fetal outcome in pregnancies complicated by intra-uterine growth retardation. Acta Obstetricia et Gynecologica Scandinavica 1987;66: 407-11.

19. Ülker K, Çeçen K, Temur İ, Gül A, Karaca M. Effects of the maternal po- sition and rest on the fetal urine production rate: a prospective study con- ducted by 3-dimensional sonography using the rotational technique (virtual organ computer-aided analysis). J Ultrasound Med 2011; 30:481- 486 .

20. Ülker K, Gül A, Çiçek M. Correlation Between the Duration of Maternal Rest in the Left Lateral Decubitus Position and the Amniotic Fluid Volume Increase. J Ultrasound Med 2012; 31:705-709

21. Marx GF, Patel S, Berman JA, Farmakides G, Schulman $H$. Umbilical blood flow velocity waveforms in different maternal positions and with epidural analgesia. Obstet Gynecol 1986; 68:61-64

22. Ryo E, Okai T, Takagi K, et al. Comparison of umbilical artery Doppler velocimetry between maternal supine position and complete left lateral position in predicting obstetric complications. Ultrasound Obstet Gynecol 1998; $11: 415-418$.

23. Purcell KJ, Schembri M, Telles TL, Fujimoto VY, Cedars MI. Bed rest after embryo transfer: a randomized controlled trial. Fertility and Sterility 2007;87(6):1322-6. [PUBMED: 17362946]

24. Kovacevich GJ, Gaich SA, Lavin JP, Hopkins MP, Crane SS, Stewart J, et al.The prevalence of thromboembolic events among women with extended bed rest prescribed as part of the treatment for premature labor or preterm premature rupture of membranes. American Journal of Obstetrics and Gynecology 2000;182(5):1089-92.

25. Maloni JA, Chance B, Zhang C, Cohen AW, Betts D, Gange SJ. Physical and psychosocial side effects of antepartum hospital bed rest. Nursing Research 1993;42(4):197-203.

26. Maloni JA, Kane JH, Suen LJ, Wang KK. Dysphoria among high-risk pregnant hospitalized women on bed rest: a longitudinal study. Nursing Research 2002;51(2):92-9.

27. Gupton A, Heaman M, Ashcroft T. Bed rest from the perspective of the high-risk pregnant woman. Journal of Obstetric, Gynecologic and Neonatal Nursing 1997;26(4): 423-30.

28. Schroeder C. Women' s experience of bed rest in high-risk pregnancy. Image - The Journal of Nursing Scholarship 1996; 28(3):253-8.

29. Allen C, Glasziou P, Del Mar C. Bed rest: a potentially harmful treatment needing more careful evaluation. Lancet 1999;354:1229-33.

30. Trudel G, Payne M, Mädler B, Ramachandran N, Lecompte $\mathrm{M}$, Wade $\mathrm{C}$ et al. Bone marrow fat accumulation after 60 days of bed rest persisted 1 year after activities were resumed along with hemopoietic stimulation: the Women International Space Simulation for Exploration study, J Appl Physiol 2009;107: 540-8 